\title{
The GUP Effect on Tunneling of Massive Vector Bosons from The 2+1 Dimensional Black Hole
}

\author{
Ganim Gecim and Yusuf Sucu (iD \\ Department of Physics, Faculty of Science, Akdeniz University, 07058 Antalya, Turkey \\ Correspondence should be addressed to Yusuf Sucu; ysucu@akdeniz.edu.tr
}

Received 2 February 2018; Revised 7 April 2018; Accepted 15 May 2018; Published 25 June 2018

Academic Editor: Ricardo G. Felipe

Copyright (C) 2018 Ganim Gecim and Yusuf Sucu. This is an open access article distributed under the Creative Commons Attribution License, which permits unrestricted use, distribution, and reproduction in any medium, provided the original work is properly cited. The publication of this article was funded by $\mathrm{SCOAP}^{3}$.

In this study, the Generalized Uncertainty Principle (GUP) effect on the Hawking radiation formed by tunneling of a massive vector boson particle from the $2+1$ dimensional new-type black hole was investigated. We used modified massive vector boson equation based on the GUP. Then, the Hamilton-Jacobi quantum tunneling approach was used to work out the tunneling probability of the massive vector boson particle and Hawking temperature of the black hole. Due to the GUP effect, the modified Hawking temperature was found to depend on the black hole properties, on the $\mathrm{AdS}_{3}$ radius, and on the energy, mass, and total angular momentum of the tunneling massive vector boson. In the light of these results, we also observed that modified Hawking temperature increases by the total angular momentum of the particle while it decreases by the energy and mass of the particle and the graviton mass. Also, in the context of the GUP, we see that the Hawking temperature due to the tunneling massive vector boson is completely different from both that of the spin-0 scalar and that of the spin-1/2 Dirac particles obtained in the previous study. We also calculate the heat capacity of the black hole using the modified Hawking temperature and then discuss influence of the GUP on the stability of the black hole.

\section{Introduction}

It was theoretically proved by Hawking that a black hole can emit thermal radiation as a result of quantum tunneling process of the particles created by the quantum fluctuation near the event horizon [1-4]. This suggestion has an important influence on understanding the quantization of black hole and gravity $[5,6]$. Since the Hawking's discovery, many different methods were developed to calculate black hole radiation, known as Hawking radiation, in the literature. For instance, the Hamilton-Jacobi approach is an effective way to study the Hawking radiation as a quantum tunneling process of particles from black holes. In this context, Hawking radiation is seen to be extensively investigated in the literature in the context of quantum tunneling of a point-like elementary particle [7-34]. These studies show that Hawking radiation of a black hole must be completely independent from the mass, total angular momentum, energy, and charge of a tunneling point particle.

On the other hand, alternative approaches about quantum gravity predict the presence of a minimal observable length in Planck scale [35-42]. The existence of such length leads to the GUP, a generalization of the standard Heisenberg uncertainty principle [36, 43-45]. Effects of the GUP on the standard quantum mechanical problems $[46,47]$, on the early and late times of the universe [48-51], and on thermodynamic properties of black holes [52-54] have been extensively discussed in the literature. To calculate the modified Hawking radiation in the GUP framework by HamiltonJacobi approach, Klein-Gordon equation for spin-0 particle, Dirac equation for spin-1/2 particle, and massive $W^{+}$-boson equation for spin-1 particle are modified [55-57]. It shows that the modified Hawking temperature depends not only on properties of black hole, but also on the properties of tunneling particle, such as mass, angular (orbital + spin) momentum, energy, charge [58-71]. Furthermore, it has been proved that the spin- 0 and spin- $1 / 2$ particles tunnel through the horizon of the $2+1$ dimensional black holes in the same way in the absence of the GUP effect. On the other hand, they tunnel differently in the presence of the GUP effect $[72,73]$. Therefore, our plan was to figure out the effect of the GUP on the Hawking temperature of the $2+1$ dimensional 
new-type black hole by using process of tunneling of the massive spin-1 particle described by the relativistic equation derived from the quantization of classical zitterbewegung model $[74,75]$. This will provide a comparison of the results of the tunneling process of the different particles types from the new-type black hole under conditions with and without the GUP effect $[32-34,73]$. The stability of the black hole will also be discussed in the context of the GUP.

The paper is organized as follows: in the following section, we modify the massive vector boson equation in the framework of the GUP. After that, in Section 3, we introduce the new-type black hole and then calculate its modified Hawking temperature by using the HamiltonJacobi approach. Moreover, using the modified Hawking temperature, the heat capacity of the black hole is calculated for analyzing the stability of the black hole. In Section 4, we summarize the results.

\section{Modified Vector Boson Equation}

In a curved space-time background, the massive vector boson equation can be written as in [74]

$$
i \beta^{\mu}(x)\left[\partial_{\mu}-\Sigma_{\mu}(x)\right] \Psi(x)=\frac{m_{0}}{\hbar} \Psi(x)
$$

where the $m_{0}$ and $\Psi(x)=\left(\psi_{+}, \psi_{0}, \psi_{0}, \psi_{-}\right)^{T}$ are the mass and the wave function of the massive vector boson, respectively $[74,76-84] . \beta^{\mu}(x)$ and $\Sigma_{\mu}(x)$ are Kemmer matrices in $2+1$ dimensional curved space-time and spin connection coefficients, respectively, and their expressions are given as

$$
\begin{aligned}
& \beta^{\mu}(x)=\bar{\sigma}^{\mu}(x) \otimes I+I \otimes \bar{\sigma}^{\mu}(x) \\
& \Sigma_{\mu}(x)=\Gamma_{\mu}(x) \otimes I+I \otimes \Gamma_{\mu}(x),
\end{aligned}
$$

in terms of the Dirac matrices, $\bar{\sigma}^{\mu}(x)$, and spin connections for Dirac particle, $\Gamma_{\mu}(x)$ [85].

To investigate the quantum gravity effect on the tunneling process of the massive vector boson, (1) has to be rewritten in the framework of the GUP. The simplest and well-known form of the GUP relation is given as follows [86-89]:

$$
\Delta x \Delta p \geq \frac{h}{2}\left[1+\alpha(\Delta p)^{2}\right]
$$

where $\alpha=\alpha_{0} / M_{p}^{2}$ with Planck mass, $M_{p}$, and a dimensionless parameter, $\alpha_{0}$. Moreover, (3) can be derived by using the modified Heisenberg algebra relation given as [86-89]

$$
\left[x_{i}, p_{j}\right]=i \hbar \delta_{i j}\left[1+\alpha p_{0 i}^{2}\right]
$$

with modified position, $x_{i}$, and modified momentum $p_{j}$ operators given in terms of the standard position, $x_{0 i}$, and momentum operators, $p_{0 j}$,

$$
\begin{aligned}
& x_{i}=x_{0 i}, \\
& p_{i}=p_{0 i}\left(1+\alpha p_{0}^{2}\right),
\end{aligned}
$$

respectively, and $p_{0}^{2}=p_{0 j} p_{0}^{j}$ [61]. On the other hand, the modified energy relation is given by the following form [56, $64,87,88]$ :

$$
\widetilde{E}=E\left(1-\alpha E^{2}\right)=E\left[1-\alpha\left(p^{2}+m_{0}^{2}\right)\right],
$$

with the energy mass shell condition, $E^{2}=p^{2}+m_{0}^{2}$. In addition, the square of the momentum operator can be expressed after neglecting the higher order terms of the $\alpha$ parameter as follows [89]:

$$
p^{2}=p_{i} p^{i} \simeq-\hbar^{2}\left[\partial_{i} \partial^{i}-2 \alpha\left(\partial_{j} \partial^{j}\right)\left(\partial_{i} \partial^{i}\right)\right]
$$

Including (5), (6), and (7), the modified massive vector boson equation can be written as follows:

$$
\begin{aligned}
& \left(i \beta^{i}(x) \partial_{i}-i \beta^{\mu}(x) \Sigma_{\mu}-\frac{m_{0}}{\hbar}\right)\left(1+\alpha \hbar^{2} \partial_{j} \partial^{j}-\alpha m_{0}^{2}\right) \widetilde{\Psi} \\
& +i \beta^{0}(x) \partial_{0} \widetilde{\Psi}=0,
\end{aligned}
$$

or in its explicit form:

$$
\begin{aligned}
i \beta^{0} \partial_{0} \widetilde{\Psi}+\left[i \beta^{i}\left(1-\alpha m_{0}^{2}\right) \partial_{i}+i \alpha \hbar^{2} \beta^{i} \partial_{i}\left(\partial_{j} \partial^{j}\right)\right] \widetilde{\Psi} \\
-\frac{m_{0}}{\hbar}\left(1+\alpha \hbar^{2} \partial_{j} \partial^{j}-\alpha m_{0}^{2}\right) \widetilde{\Psi} \\
-i \beta^{\mu} \Sigma_{\mu}\left(1+\alpha \hbar^{2} \partial_{j} \partial^{j}-\alpha m_{0}^{2}\right) \widetilde{\Psi}=0
\end{aligned}
$$

where the $\widetilde{\Psi}$ is the modified wave function of a vector boson.

\section{Tunneling of Vector Boson Particle from New-Type Black Hole}

In the framework of the quantum gravity, several studies have been carried out with a focus on $2+1$ dimensional theories (i.e., a toy model) [90-93]. The New Massive Gravity is just one of them [94]. It has topologically a graviton with mass [94-96]. This theory provides black hole solutions. For example, the new-type black hole, which is asymptotically anti-Sitter, static, and circular symmetric, is a solution to field equations of the theory. Also, the new-type black hole is conformally flat, and therefore it corresponds to a solution of the $2+1$ dimensional conformal gravity theory $[97,98]$. The space-time metric of the black hole is given as

$$
d s^{2}=L^{2}\left[f(r) d t^{2}-\frac{1}{f(r)} d r^{2}-r^{2} d \phi^{2}\right],
$$

where $f(r)=r^{2}+b r+c$ and $b$ and $c$ are two constant parameters related to the "gravitational hair" and "mass", respectively [97-100]. $L$ is the $\mathrm{AdS}_{3}$ radius defined as $L^{2}=1 / 2 m^{2}=1 / 2 \Lambda$ in terms of the graviton mass, $m$ (or the cosmological constant, $\Lambda)$. The black hole has an outer and an inner horizon located at $r_{+}=(1 / 2)\left(-b+\sqrt{b^{2}-4 c}\right)$ and $r_{-}=(1 / 2)\left(-b-\sqrt{b^{2}-4 c}\right)$, respectively. Also, it has a curvature singularity at $r=0$ when $b \neq 0$. This singularity is always surrounded by an event horizon. Also, the signatures of the $b$ and $c$ parameters play important role in the mathematical and physical properties of 
the black hole $[97,99]$. The horizons coincide with each other when $b^{2}=4 c$; i.e., the black hole becomes extremal. Moreover, when $b=0$ and $c<0$, the black hole is reduced to the static Banados-Teitelboim-Zanelli (BTZ) black hole [97, 99].

In this work, the effect of the GUP on the Hawking temperature of the black hole was investigated by tunneling of the massive vector boson particle. In this investigation, the Kemmer matrices and spin connection coefficients from [34] were employed. Inserting the following ansatz for the modified wave function [74],

$$
\widetilde{\Psi}(x)=\exp \left(\frac{i}{\hbar} S(t, r, \phi)\right)\left(\begin{array}{c}
A(t, r, \phi) \\
B(t, r, \phi) \\
B(t, r, \phi) \\
D(t, r, \phi)
\end{array}\right),
$$

in (9) and neglecting the terms with $\hbar$, we obtain the following three decoupled differential equations:

$$
\begin{aligned}
& B\left[i \frac{L^{2}}{r} \frac{\partial S}{\partial \phi}+i \alpha \frac{1}{r^{3}}\left(\frac{\partial S}{\partial \phi}\right)^{3}-\alpha \frac{\sqrt{f}}{r^{2}}\left(\frac{\partial S}{\partial \phi}\right)^{2}\left(\frac{\partial S}{\partial r}\right)\right. \\
& \left.+\alpha L^{2} m_{0}^{2} f \frac{\partial S}{\partial r}-L^{2} \sqrt{f} \frac{\partial S}{\partial r}\right] \\
& +B\left[i \alpha \frac{f}{r}\left(\frac{\partial S}{\partial \phi}\right)\left(\frac{\partial S}{\partial r}\right)^{2}-\alpha f \sqrt{f}\left(\frac{\partial S}{\partial r}\right)^{3}\right. \\
& \left.-i \alpha \frac{m_{0}^{2} L^{2}}{r} \frac{\partial S}{\partial \phi}\right]+A\left[i \alpha \frac{L m_{0}}{2 r^{2}}\left(\frac{\partial S}{\partial \phi}\right)^{2}\right. \\
& +i \alpha \frac{L m_{0} f}{2}\left(\frac{\partial S}{\partial r}\right)^{2}+i L^{3} \frac{m_{0}\left(1-\alpha m_{0}^{2}\right)}{2} \\
& \left.+i \frac{L^{2}}{\sqrt{f}} \frac{\partial S}{\partial t}\right]=0 \\
& A\left[-L^{2} \sqrt{f} \frac{\partial S}{\partial r}-i \alpha \frac{f}{r}\left(\frac{\partial S}{\partial \phi}\right)\left(\frac{\partial S}{\partial r}\right)^{2}+i \alpha \frac{m_{0}^{2} L^{2}}{r} \frac{\partial S}{\partial \phi}\right. \\
& \left.-\alpha f \sqrt{f}\left(\frac{\partial S}{\partial r}\right)^{3}-i \alpha \frac{1}{r^{3}}\left(\frac{\partial S}{\partial \phi}\right)^{3}\right]+A\left[-i \frac{L^{2}}{r} \frac{\partial S}{\partial \phi}\right. \\
& \left.-\alpha \frac{\sqrt{f}}{r^{2}}\left(\frac{\partial S}{\partial \phi}\right)^{2}\left(\frac{\partial S}{\partial r}\right)+\alpha L^{2} \sqrt{f} m_{0}^{2} \frac{\partial S}{\partial r}\right] \\
& +D\left[i \frac{L^{2}}{r} \frac{\partial S}{\partial \phi}-i \alpha \frac{m_{0}^{2} L^{2}}{r} \frac{\partial S}{\partial \phi}+i \alpha \frac{f}{r}\left(\frac{\partial S}{\partial \phi}\right)\left(\frac{\partial S}{\partial r}\right)^{2}\right. \\
& \left.-L^{2} \sqrt{f} \frac{\partial S}{\partial r}-\alpha f \sqrt{f}\left(\frac{\partial S}{\partial r}\right)^{3}\right]+D\left[\alpha L^{2} m_{0}^{2} f \frac{\partial S}{\partial r}\right. \\
& \left.+i \alpha \frac{1}{r^{3}}\left(\frac{\partial S}{\partial \phi}\right)^{3}-\alpha \frac{\sqrt{f}}{r^{2}}\left(\frac{\partial S}{\partial \phi}\right)^{2}\left(\frac{\partial S}{\partial r}\right)\right] \\
& +B\left[i L^{3} m_{0}\left(1-\alpha m_{0}\right)+i \alpha L m_{0} f\left(\frac{\partial S}{\partial r}\right)^{2}\right.
\end{aligned}
$$

$$
\begin{aligned}
& \left.+i \alpha \frac{m_{0} L}{r^{2}}\left(\frac{\partial S}{\partial \phi}\right)^{2}\right]=0 \\
& B\left[-L^{2} \sqrt{f} \frac{\partial S}{\partial r}-i \frac{L^{2}}{r} \frac{\partial S}{\partial \phi}-\alpha \frac{\sqrt{f}}{r^{2}}\left(\frac{\partial S}{\partial \phi}\right)^{2}\left(\frac{\partial S}{\partial r}\right)\right. \\
& \left.+\alpha L^{2} m_{0}^{2} \frac{\partial S}{\partial r}-i \alpha \frac{1}{r^{3}}\left(\frac{\partial S}{\partial \phi}\right)^{3}\right] \\
& +B\left[-i \alpha \frac{f}{r}\left(\frac{\partial S}{\partial \phi}\right)\left(\frac{\partial S}{\partial r}\right)^{2}+i \alpha \frac{m_{0}^{2} L^{2}}{r} \frac{\partial S}{\partial \phi}\right. \\
& \left.+\alpha f \sqrt{f}\left(\frac{\partial S}{\partial r}\right)^{3}\right]+D\left[i L^{3} \frac{m_{0}\left(1-\alpha m_{0}^{2}\right)}{2}\right. \\
& \left.+i \alpha \frac{L m_{0}}{2 r^{2}}\left(\frac{\partial S}{\partial \phi}\right)^{2}+i \alpha \frac{L m_{0} f}{2}\left(\frac{\partial S}{\partial r}\right)^{2}-i \frac{L^{2}}{\sqrt{f}} \frac{\partial S}{\partial t}\right] \\
& \quad=0 .
\end{aligned}
$$

where $A(t, r, \phi), B(t, r, \phi)$, and $D(t, r, \phi)$ are functions of the space-time coordinates and $S(t, r, \phi)$ is the particle trajectory known as classical action. The nontrivial solution was found by the condition put on the determinants of the coefficients. Accordingly, we get the modified Hamilton-Jacobi equation as

$$
\begin{gathered}
\frac{1}{f}\left(\frac{\partial S}{\partial t}\right)^{2}-\frac{1}{r^{2}}\left(\frac{\partial S}{\partial \phi}\right)^{2}-f\left(\frac{\partial S}{\partial r}\right)^{2}-\frac{m_{0}^{2} L^{2}}{4} \\
+\alpha\left[\frac{9 m_{0}^{2} f}{4}\left(\frac{\partial S}{\partial r}\right)^{2}+\frac{1}{L^{2}}\left(\frac{\partial S}{\partial t}\right)^{2}\left(\frac{\partial S}{\partial r}\right)^{2}\right] \\
+\alpha\left[-\frac{6 f}{L^{2} r^{2}}\left(\frac{\partial S}{\partial r}\right)^{2}\left(\frac{\partial S}{\partial \phi}\right)^{2}+\frac{9 m_{0}^{2}}{4 r^{2}}\left(\frac{\partial S}{\partial \phi}\right)^{2}\right. \\
\left.+\frac{3 m_{0}^{4} L^{2}}{4}-\frac{3 f^{2}}{L^{2}}\left(\frac{\partial S}{\partial r}\right)^{4}-\frac{3}{L^{2} r^{4}}\left(\frac{\partial S}{\partial \phi}\right)^{4}\right] \\
+\alpha\left[\frac{1}{L^{2} r^{2} f}\left(\frac{\partial S}{\partial t}\right)^{2}\left(\frac{\partial S}{\partial \phi}\right)^{2}-\frac{m_{0}^{2}}{f}\left(\frac{\partial S}{\partial t}\right)^{2}\right]=0
\end{gathered}
$$

Using the separation of variables method, the action function, $S(t, r, \phi)$, is decomposed into its components as follows:

$$
S(t, r, \phi)=-E t+j \phi+K(r)+C,
$$

where $E$ and $j$ are the energy and total angular momentum of the particle, respectively, $K(r)=K_{0}(r)+\alpha K_{1}(r)$ [65], and the $C$ is a complex constant. Inserting (14) into (13), we get the radial trajectory function, $K(r)$, of the particle as follows:

$$
K_{ \pm}(r)= \pm \int \frac{\sqrt{E^{2}-f\left(m_{0}^{2}+j^{2} / r^{2}\right)}}{f}[1+\alpha \chi] d r
$$

where $\chi$ is

$$
\chi=\frac{1}{4 L^{2} f}\left(\frac{5 E^{2} L^{2} m_{0}^{2} f-4 E^{4}}{E^{2}-f\left(m_{0}^{2}+j^{2} / r^{2}\right)}\right) .
$$


Here, $K_{+}(r)$ and $K_{-}(r)$ represent the radial trajectories of the outgoing and incoming particles on the outer horizon, respectively. Therefore, the integration in (15) is calculated as

$$
K_{ \pm}(r)= \pm i \frac{\pi E}{\left(r_{+}-r_{-}\right)}[1+\alpha \Omega] .
$$

Here $\Omega$ is

$$
\Omega=\frac{\left(r_{+}-r_{-}\right)^{2}\left(9 L^{2} m_{0}^{2} r_{+}^{2}-4 j^{2}\right)+16 E^{2} r_{+}^{2}}{8 L^{2} r_{+}^{2}\left(r_{+}-r_{-}\right)^{2}} .
$$

Accordingly, the outgoing and ingoing probabilities of the massive vector boson particles crossing the outer horizon are given by

$$
\begin{aligned}
P_{\text {out }} & =\exp \left[-\frac{2}{\hbar} \operatorname{Im} K_{+}(r)\right] \\
P_{\text {in }} & =\exp \left[-\frac{2}{\hbar} \operatorname{Im} K_{-}(r)\right],
\end{aligned}
$$

respectively. Furthermore, the total tunneling probability of the massive vector boson particle crossing the horizon is expressed as [20]

$$
\Gamma=e^{-(2 / \hbar) I m S(t, r, \phi)}=\frac{P_{\text {out }}}{P_{\text {in }}} .
$$

Using (19) and (20) and the fact that $\operatorname{Im} K_{+}(r)=-\operatorname{Im} K_{-}(r)$, the total tunneling probability is obtained as follows:

$$
\begin{aligned}
\Gamma & =\exp \left[-\frac{4}{\hbar} \operatorname{Im} K_{+}(r)\right] \\
& =\exp \left\{-\frac{4 \pi E}{\left(r_{+}-r_{-}\right)}[1+\alpha \Omega]\right\} .
\end{aligned}
$$

On the other hand, the tunneling probability can be expressed in terms of Boltzmann factor as

$$
\Gamma=e^{-(2 / \hbar) \operatorname{Im} S}=e^{-\beta E},
$$

where $\beta$ is the inverse of temperature. Finally, the modified Hawking temperature becomes

$$
T_{H}^{\prime}=\hbar \frac{\left(r_{+}-r_{-}\right)}{4 \pi}[1+\alpha \Omega]^{-1} .
$$

If we expand the $T_{H}^{\prime}$ in terms of the $\alpha$ powers and neglect the higher order terms, the modified Hawking temperature of the new-type black hole becomes as follows:

$$
T_{H}^{\prime}=T_{H}[1-\alpha \Omega]
$$

where the $T_{H}=\hbar\left(\left(r_{+}-r_{-}\right) / 4 \pi\right)$ is the standard Hawking temperature of the black hole. From the $T_{H}^{\prime}$ expression, we see that the modified Hawking temperature can be related not only to the mass of the black hole, but also to the $\mathrm{AdS}_{3}$ radius $L$ (and, hence, to the graviton mass) and the properties of the tunneled massive vector boson, such as angular momentum, energy, and mass. In addition, this result indicates that the modified Hawking temperature caused by tunneling vector boson particle is completely different from that of both scalar and Dirac particles [73]. Therefore, in the framework of the GUP, one can say that massive spin-0 scalar, massive spin-1/2 Dirac, and massive spin-1 vector boson particles probe the black hole in different manners. On the other hand, in the case of $\alpha=0$, the modified Hawking temperature is reduced to the standard temperature obtained by quantum tunneling process of the point particles with spin-0, spin-1/2, and spin-1, respectively $[32,34]$.

The local stability of a black hole can be analyzed by its heat capacity [101]. The positive heat capacity means that black hole is locally stable; otherwise it is unstable [102-104]. The modified heat capacity of a black hole can be calculated from the relation

$$
C^{\prime}=\frac{\partial M}{\partial T_{H}^{\prime}}
$$

where $T_{H}^{\prime}$ is the modified Hawking temperature given in (23) and $M$ is mass of the black hole given as [99]

$$
M=\frac{\left(r_{+}^{2}-r_{-}^{2}\right)}{16 G}
$$

with gravitational constant $G$. Hence, using (23) and (26), the modified heat capacity of the black hole is calculated as

$$
\begin{aligned}
C^{\prime} & \\
= & \frac{\pi\left(r_{+}-r_{-}\right)}{2 \hbar G} \\
& +\alpha \pi \frac{\left[9 L^{2} m_{0}^{2} r_{+}^{5}+9 L^{2} m_{0}^{2} r_{+}^{3} r_{-}^{2}+4 j^{2} r_{+}^{3}+20 r_{+} j^{2} r_{-}^{2}\right]}{16 \hbar G L^{2} r_{+}^{3}\left(r_{+}-r_{-}\right)} \\
& -\alpha \pi \frac{\left[18 L^{2} m_{0}^{2} r_{+}^{4} r_{-}+16 E^{2} r_{+}^{3}+16 j^{2} r_{+}^{2} r_{-}+8 j^{2} r_{+}^{3}\right]}{16 \hbar G L^{2} r_{+}^{3}\left(r_{+}-r_{-}\right)}
\end{aligned}
$$

or

$$
C^{\prime}=\frac{\pi(\mathscr{A}-\mathscr{B})}{16 \hbar G L^{2} r_{+}^{3}\left(r_{+}-r_{-}\right)},
$$

where the abbreviations $\mathscr{A}$ and $\mathscr{B}$ are

$$
\begin{aligned}
\mathscr{A}= & 8 L^{2} r_{+}^{5}+8 L^{2} r_{+}^{3} r_{-}^{2}+9 \alpha L^{2} m_{0}^{2} r_{+}^{5}+4 \alpha j^{2} r_{+}^{3} \\
& +9 \alpha L^{2} m_{0}^{2} r_{+}^{3} r_{-}^{2}+20 \alpha j^{2} r_{+} r_{-}^{2}, \\
\mathscr{B}= & 16 L^{2} r_{+}^{4} r_{-}+18 \alpha L^{2} m_{0}^{2} r_{+}^{4} r_{-}+16 \alpha j^{2} r_{-} r_{+}^{2} \\
& +16 \alpha E^{2} r_{+}^{3}+8 \alpha j^{2} r_{-}^{3},
\end{aligned}
$$

respectively. The points where the heat capacity vanishes or diverges represent the phase transition points where the black hole transfers from an unstable state to a stable state. The points, where the heat capacity is vanished correspond to a first-order phase transition while the diverged points correspond to a second-order phase transition. According to 
(28), the modified heat capacity diverges at point $r_{+}=r_{-}$. This indicates that the black hole is stabilized by passing through a second-order phase transition. The modified heat capacity is positive for $r_{+}>r_{-}$. Hence in this region, the black hole is said to be locally stable. However, for $r_{+}<\left|r_{-}\right|$, the black hole is locally unstable. On the other hand, for $r_{+}>r_{-}$, the modified heat capacity vanishes at point $\mathscr{A}=\mathscr{B}$. This case indicates that the black hole undergoes a first-order phase transition to become stable. In the absence of the GUP effect the heat capacity becomes as

$$
C=\frac{\pi\left(r_{+}-r_{-}\right)}{2 \hbar G}
$$

According to this, it can be said that the heat capacity vanishes at point $r_{+}=r_{-}$whereas there is no point where it diverges. This indicates that the black hole has become stable through only the first-order phase transition.

\section{Summary and Conclusion}

In this study, we investigate the quantum gravity effect on the tunneling massive vector boson from the new-type black hole in the context of the $2+1$ dimensional New Massive Gravity. For this, using the GUP relations, we first modified the massive vector boson equation. Then, using the Hamilton-Jacobi approach, the tunneling probability of the massive vector particle was derived, and, subsequently, the modified Hawking temperature of the black hole was calculated. We also found that the modified Hawking temperature not only depends on the black hole's properties, but also depends on the emitted spin-1 vector boson's mass, energy, and total angular momentum. It is also worth mentioning that the modified Hawking temperature is seen to be dependent on the mass of graviton in this context. As can be seen from (24), the Hawking temperature increases by the total angular momentum of the tunneled particle while it decreases by the energy and mass of the tunneled particle and the graviton mass.

In addition, according to (24), we can summarize some important results as follows:

(i) If $9 r_{+}^{2}\left(r_{+}-r_{-}\right)^{2}\left(m_{0}^{2} / m^{2}\right)+16 E^{2} r_{+}^{2}>4 j^{2}\left(r_{+}-r_{-}\right)^{2}$, the modified Hawking temperature of the tunneling vector boson is lower than the standard temperature. However, when $9 r_{+}^{2}\left(r_{+}-r_{-}\right)^{2}\left(m_{0}^{2} / m^{2}\right)+16 E^{2} r_{+}^{2}<$ $4 j^{2}\left(r_{+}-r_{-}\right)^{2}$, the corrected temperature is higher than the standard temperature. If $9 r_{+}^{2}\left(r_{+}-r_{-}\right)^{2}\left(m_{0}^{2} / m^{2}\right)+$ $16 E^{2} r_{+}^{2}=4 j^{2}\left(r_{+}-r_{-}\right)^{2}$, then the contribution of the GUP effect is canceled, and the modified temperature of the tunneling vector boson reduces to the standard temperature.

(ii) As described previously, the new-type black hole is reduced to the static BTZ black hole in the case of $b=0$ and $c<0$. Hence, the modified Hawking temperature of the static BTZ black hole under the GUP effect is

$$
T_{H}^{\prime}=T_{H}\left[1-\alpha m^{2} \frac{\left[\left(9 m_{0}^{2} / 2 m^{2}\right)|c|-4 j^{2}\right]+4 E^{2}}{4|c|}\right],
$$

where $r_{+}=-r_{-}=\sqrt{|c|}$ is used and $T_{H}=\hbar(\sqrt{|c|} / 2 \pi)$ is the standard Hawking temperature of the static BTZ black hole in the context of the $2+1$ dimensional New Massive Gravity theory [32]. In this case, the modified Hawking temperature is higher than the standard Hawking temperature when $4 E^{2}+\left(9 m_{0}^{2} / 2 m^{2}\right)|c|<$ $4 j^{2}$. On the other hand, as $4 E^{2}+\left(9 m_{0}^{2} / 2 m^{2}\right)|c|>4 j^{2}$, the modified Hawking temperature is lower than the standard Hawking temperature.

(iii) In the absence of the quantum gravity effect, i.e., $\alpha=0$, the modified Hawking temperature is reduced to the standard temperature obtained by quantum tunneling of the massive spin- 0 , spin- $1 / 2$, and spin-1 point particles $[32,34]$.

In this study we also consider the local stability of the black hole under the GUP effect. At that case, we observe that in the absence of quantum gravity effect, the black hole undergoes first-order phase transition only, but, in the presence of the quantum gravity effect, it undergoes the firstand second-order phase transitions to become stable.

\section{Data Availability}

No data were used to support this study.

\section{Conflicts of Interest}

The authors declare that there are no conflicts of interest regarding the publication of this paper.

\section{Acknowledgments}

This work was supported by Akdeniz University Scientific Research Projects Unit, and the Scientific and Technological Research Council of Turkey (TUBITAK) 1002-QSP (Project no. 116F329).

\section{References}

[1] S. W. Hawking, "Black hole explosions?" Nature, vol. 248, pp. 30-31, 1974.

[2] S. W. Hawking, "Particle creation by black holes," Communications in Mathematical Physics, vol. 43, no. 3, pp. 199-220, 1975.

[3] S. W. Hawking, "Black holes and thermodynamics," Physical Review D: Particles, Fields, Gravitation and Cosmology, vol. 13, no. 2, pp. 191-197, 1976.

[4] J. M. Bardeen, B. Carter, and S. W. Hawking, "The four laws of black hole mechanics," Communications in Mathematical Physics, vol. 31, pp. 161-170, 1973.

[5] S. P. Robinson and F. Wilczek, "Relationship between Hawking radiation and gravitational anomalies," Physical Review Letters, vol. 95, Article ID 011303, 2005.

[6] S. B. Giddings, "High-energy black hole production," AIP Conference Proceedings, vol. 957, no. 1, p. 69, 2007.

[7] P. Kraus and F. Wilczek, "Effect of self-interaction on charged black hole radiance," Nuclear Physics B, vol. 437, no. 1, pp. 231242, 1995.

[8] P. Kraus and F. Wilczek, "Self-interaction correction to black hole radiance," Nuclear Physics B, vol. 433, no. 2, pp. 403-420, 1995. 
[9] M. K. Parikh and F. Wilczek, "Hawking radiation as tunneling," Physical Review Letters, vol. 85, no. 24, pp. 5042-5045, 2000.

[10] R. Kerner and R. B. Mann, "Tunnelling, temperature, and Taub-NUT black holes," Physical Review D: Particles, Fields, Gravitation and Cosmology, vol. 73, Article ID 104010, 2006.

[11] R. Kerner and R. B. Mann, "Fermions tunnelling from black holes," Classical and Quantum Gravity, vol. 25, no. 9, Article ID 095014, 2008.

[12] D. Y. Chen, Q. Q. Jiang, and X. T. Zu, "Fermions tunnelling from the charged dilatonic black holes," Classical and Quantum Gravity, vol. 25, Article ID 205022, 2008.

[13] J. Zhang and Z. Zhao, "Charged particles' tunnelling from the Kerr-Newman black hole," Physics Letters B, vol. 638, no. 2-3, pp. 110-113, 2006.

[14] J. Huang and W. Liu, "Fermion tunneling from an axis-symmetric black hole beyond the semiclassical approximation," International Journal of Theoretical Physics, vol. 49, no. 10, pp. 2621-2629, 2010.

[15] X. X. Zeng and Q. Li, "On particles tunneling from the TaubNUT-AdS black hole," Chinese Physics B, vol. 18, no. 11, pp. 47164720, 2009.

[16] R. R. Criscienzo and L. L. Vanzo, "Fermion tunneling from dynamical horizons," Europhysics Letters, vol. 82, no. 6, Article ID 60001, 2008.

[17] D. Y. Chen, Q. Q. Jian, and X. T. Zu, "Hawking radiation of Dirac particles via tunnelling from rotating black holes in de Sitter spaces," Physics Letters B, vol. 665, no. 2-3, pp. 106-110, 2008.

[18] Q. Q. Jian, “Dirac particle tunneling from black rings," Physical Review D: Particles, Fields, Gravitation and Cosmology, vol. 78, Article ID 044009, 2008.

[19] R. Li, J.-K. Zhao, and X.-H. Wu, "Tunneling radiation of massive vector bosons from dilaton black holes," Communications in Theoretical Physics, vol. 66, no. 1, pp. 77-83, 2016.

[20] R. Li and J.-R. Ren, "Dirac particles tunneling from BTZ black hole," Physics Letters B, vol. 661, no. 5, pp. 370-372, 2008.

[21] H. Li, S. Yang, Q. Jiang, and D. Qi, “Charged particle's tunneling radiation from the charged BTZ black hole," Physics Letters B, vol. 641, no. 2, pp. 139-144, 2006.

[22] S. I. Kruglov, "Black hole radiation of spin-1 particles in $(1+2)$ dimensions," Modern Physics Letters A, vol. 29, no. 39, Article ID 1450203, 2014.

[23] H. Gursel and I. Sakalli, "Hawking radiation of massive vector particles from a warped $\mathrm{AdS}_{3}$ black hole," Canadian Journal of Physics, vol. 94, no. 2, pp. 147-149, 2016.

[24] I. Sakalli and A. Ovgun, "Hawking radiation of spin-1 particles from a three-dimensional rotating hairy black hole," Journal of Experimental and Theoretical Physics, vol. 121, no. 3, pp. 404407, 2015.

[25] G.-R. Chen, S. Zhou, and Y.-C. Huang, "Vector particles tunneling from BTZ black holes," International Journal of Modern Physics D, vol. 24, no. 1, Article ID 1550005, 2015.

[26] G. R. Chen and Y. C. Huang, "Hawking radiation of vector particles as tunneling from the apparent horizon of Vaidya black holes," International Journal of Modern Physics A, vol. 30, no. 15, Article ID 1550083, 2015.

[27] X.-Q. Li and G.-R. Chen, "Massive vector particles tunneling from Kerr and Kerr-Newman black holes," Physics Letters B, vol. 751, pp. 34-38, 2015.

[28] I. Sakalli and A. Övgün, "Quantum tunneling of massive spin1 particles from non-stationary metrics," General Relativity and Gravitation, vol. 48, article 1, 2016.
[29] G.-R. Chen, S. Zhou, and Y.-C. Huang, "Vector particles tunneling from four-dimensional Schwarzschild black holes," Astrophysics and Space Science, vol. 357, article 51, 2015.

[30] R. Li and J. Zhao, "Hawking radiation of massive vector particles from the linear dilaton black holes," The European Physical Journal Plus, vol. 131, article 249, 2016.

[31] I. Sakalli and H. Gursel, "Quantum tunneling from rotating black holes with scalar hair in three dimensions," The European Physical Journal C, vol. 76, no. 6, article 318, 2016.

[32] G. Gecim and Y. Sucu, "Tunnelling of relativistic particles from new type black hole in new massive gravity," Journal of Cosmology and Astroparticle Physics, vol. 2013, no. 2, article 023, 2013.

[33] G. Gecim and Y. Sucu, "Dirac and scalar particles tunnelling from topological massive warped-AdS 3 black hole," Astrophysics and Space Science, vol. 357, article 105, 2015.

[34] G. Gecim and Y. Sucu, "Massive vector bosons tunnelled from the (2+1)-dimensional black holes," The European Physical Journal Plus, vol. 132, article 105, 2017.

[35] P. K. Townsend, "Small-scale structure of spacetime as the origin of the gravitational constant," Physical Review D: Particles, Fields, Gravitation and Cosmology, vol. 15, article 2795, 1977.

[36] D. Amati, M. Ciafaloni, and G. Veneziano, "Can spacetime be probed below the string size?" Physics Letters B, vol. 216, no. 1-2, pp. 41-47, 1989.

[37] K. Konishi, G. Paffuti, and P. Provero, "Minimum physical length and the generalized uncertainty principle in string theory," Physics Letters B, vol. 234, no. 3, pp. 276-284, 1990.

[38] L. J. Garay, "Quantum gravity and minimum length," International Journal of Modern Physics A, vol. 10, no. 2, pp. 145-166, 1995.

[39] A. Kempf and G. Mangano, "Minimal length uncertainty relation and ultraviolet regularization," Physical Review D: Particles, Fields, Gravitation and Cosmology, vol. 55, no. 12, article 7909, 1997.

[40] A. F. Ali, M. M. Khalil, and E. C. Vagenas, "Minimal length in quantum gravity and gravitational measurements," EPL (Europhysics Letters), vol. 112, no. 2, Article ID 20005, 2015.

[41] A. N. Tawfik and A. M. Diab, "A review of the generalized uncertainty principle," Reports on Progress in Physics, vol. 78, no. 12, Article ID 126001, 2015.

[42] A. Tawfik and A. Diab, "Effect of the generalized uncertainty principle on compact stars," International Journal of Modern Physics D: Gravitation, Astrophysics, Cosmology, vol. 23, no. 10, pp. 161-170, 2014.

[43] M. Maggiore, "A Generalized Uncertainty Principle in Quantum Gravity," Physics Letters B, vol. 304, no. 1-2, pp. 65-69, 1993.

[44] F. Scardigli, "Generalized uncertainty principle in quantum gravity from micro-black hole gedanken experiment," Physics Letters B, vol. 452, no. 1-2, pp. 39-44, 1999.

[45] T. Ghaneh, F. Darabi, and H. Motavalli, "Signature change by gup," International Journal of Modern Physics D, vol. 22, no. 5, Article ID 1350026, 2013.

[46] F. Brau, "Minimal length uncertainty relation and the hydrogen atom," Journal of Physics A: Mathematical and Theoretical, vol. 32, no. 44, pp. 7691-7696, 1999.

[47] P. Pedram, M. Amirfakhrian, and H. Shababi, "On the $(2+1)$ dimensional Dirac equation in a constant magnetic field with a minimal length uncertainty," International Journal of Modern Physics D, vol. 24, no. 2, Article ID 1550016, 2015. 
[48] K. Nozari, S. Akhshabi, and J. Mod, "Effects of the generalized uncertainty principle on the inflation parameters," International Journal of Modern Physics D, vol. 19, no. 5, pp. 513-521, 2010.

[49] B. Vakili, "Cosmology with minimal length uncertainty relations," International Journal of Modern Physics D, vol. 18, no. 7, pp. 1059-1071, 2009.

[50] A. F. Ali and B. Majumder, "Towards a cosmology with minimal length and maximal energy," Classical and Quantum Gravity, vol. 31, no. 21, Article ID 215007, 2014.

[51] B. Majumder, " $f(R)$ in holographic and agegraphic dark energy models and the generalized uncertainty principle," Advances in High Energy Physics, vol. 2013, Article ID 143195, pp. 1-11, 2013.

[52] J. Sadeghi and V. R. Shajiee, "Quantum tunneling from the charged non-rotating BTZ black hole with GUP," The European Physical Journal Plus, vol. 132, article 132, 2017.

[53] Y. S. Myung, Y.-W. Kim, and Y.-J. Park, "Black hole thermodynamics with generalized uncertainty principle," Physics Letters $B$, vol. 645, no. 5-6, pp. 393-397, 2007.

[54] A. F. Ali and M. Moussa, "Towards thermodynamics with generalized uncertainty principle," Advances in High Energy Physics, vol. 2014, Article ID 629148, pp. 1-7, 2014.

[55] T. K. Jana and P. Roy, "Exact solution of the Klein-Gordon equation in the presence of a minimal length," Physics Letters A, vol. 373, no. 14, pp. 1239-1241, 2009.

[56] K. Nozari and M. Karami, "Minimal length and generalized Dirac equation," Modern Physics Letters A, vol. 20, no. 40, pp. 3095-3103, 2005.

[57] X.-Q. Li, "Massive vector particles tunneling from black holes influenced by the generalized uncertainty principle," Physics Letters B, vol. 763, pp. 80-86, 2016.

[58] K. Nozari and S. Saghafi, "Natural cutoffs and quantum tunneling from black hole horizon," Journal of High Energy Physics, vol. $5,2012$.

[59] D. Chen, H. Wu, and H. Yang, "Fermion's tunnelling with effects of quantum gravity," Advances in High Energy Physics, vol. 2013, Article ID 432412, pp. 1-6, 2013.

[60] D. Y. Chen, Q. Q. Jiang, P. Wang, and H. Yang, "Remnants, fermions' tunnelling and effects of quantum gravity," Journal of High Energy Physics, vol. 176, 2013.

[61] D. Chen, H. Wu, H. Yang, and S. Yang, "Effects of quantum gravity on black holes," International Journal of Modern Physics A, vol. 29, no. 26, Article ID 1430054, 2014.

[62] X.-X. Zeng and Y. Chen, "Quantum gravity corrections to fermions' tunnelling radiation in the Taub-NUT spacetime," General Relativity and Gravitation, vol. 47, article 47, 2015.

[63] H. L. Li, Z. W. Feng, and X. T. Zu, "Quantum tunneling from high dimensional Gödel black hole," General Relativity and Gravitation, vol. 48, article 18, 2016.

[64] P. Wang, H. Yang, and S. Ying, "Quantum gravity corrections to the tunneling radiation of scalar particles," International Journal of Theoretical Physics, vol. 55, no. 5, pp. 2633-2642, 2016.

[65] Z.-Y. Liu and J.-R. Ren, "Fermions tunnelling with quantum gravity correction," Communications in Theoretical Physics, vol. 62, no. 6, pp. 819-823, 2014

[66] B. Mu, P. Wang, and H. Yang, "Minimal length effects on tunnelling from spherically symmetric black holes," Advances in High Energy Physics, vol. 2015, Article ID 898916, pp. 1-8, 2015.

[67] G. Li and X. Zu, "Scalar particles' tunneling and effect of quantum gravity," Journal of Applied Mathematics and Physics, vol. 3, no. 2, Article ID 53657, pp. 134-139, 2015.
[68] M. A. Anacleto, F. A. Brito, and E. Passos, "Quantum-corrected self-dual black hole entropy in tunneling formalism with GUP," Physics Letters B, vol. 749, pp. 181-186, 2015.

[69] $\mathrm{H}$. $\mathrm{Li}$ and X. Zu, "Black hole remnant and quantum tunnelling in three-dimensional Gödel spacetime," Astrophysics and Space Science, vol. 357, article 6, 2015.

[70] I. Sakalli, A. Övgün, and K. Jusufi, "GUP assisted Hawking radiation of rotating acoustic black holes," Astrophysics and Space Science, vol. 361, article 330, 2016.

[71] E. C. Vagenas, S. M. Alsaleh, and A. F. Ali, "GUP parameter and black-hole temperature," EPL (Europhysics Letters), vol. 120, no. 4, Article ID 40001, 2017.

[72] G. Gecim and Y. Sucu, "The GUP effect on Hawking radiation of the $2+1$ dimensional black hole," Physics Letters B, vol. 773, pp. 391-394, 2017.

[73] G. Gecim and Y. Sucu, "Quantum gravity effect on the tunneling particles from $2+1$-dimensional new-type black hole," Advances in High Energy Physics, vol. 2018, Article ID 8728564, pp. 1-7, 2018.

[74] M. Dernek, S. Gurtas Dogan, Y. Sucu, and N. Unal, "Relativistic quantum mechanical spin-1 wave equation in $2+1$ dimensional spacetime," 2017, https://arxiv.org/abs/1606.03296v2.

[75] C. Tekincay and Y. Sucu, "Photon in a cylindrical resonant cavity," Turkish Journal of Physics, vol. 42, pp. 175-182, 2018.

[76] Y. Sucu and N. Ünal, "Vector bosons in the expanding universe," The European Physical Journal C, vol. 44, no. 2, pp. 287-291, 2005.

[77] A. O. Barut, "Excited states of zitterbewegung," Physics Letters $B$, vol. 237, no. 3-4, pp. 436-439, 1990.

[78] N. Unal, "Kernel of the classical zitterbewegung," Foundations of Physics, vol. 27, no. 5, pp. 747-758, 1997.

[79] N. Ünal, "A simple model of the classical zitterbewegung: photon wave function," Foundations of Physics, vol. 27, no. 5, pp. 731-746, 1997.

[80] N. Ünal, "Path integral quantization of a spinning particle," Foundations of Physics. An International Journal Devoted to the Conceptual Bases and Fundamental Theories of Modern Physics, vol. 28, no. 5, pp. 755-762, 1998.

[81] Y. Sucu and N. Unal, International Journal of Modern Physics A, vol. 17, no. 8, pp. 1137-1147, 2002.

[82] Y. Sucu and N. Ünal, "Symmetry and integrability in the classical model of Zitterbewegung," Foundations of Physics, vol. 42, no. 8, pp. 1067-1077, 2012.

[83] L. B. Castro and A. S. de Castro, "Corroborating the equivalence between the Duffin-Kemmer-Petiau and the Klein-Gordon and Proca equations," Physical review A, vol. 90, Article ID 022101, 2014.

[84] N. Unal, "Duffin-Kemmer-Petiau equation, proca equation and Maxwells equation in 1+1 D," Concepts of Physics, vol. 2, pp. 273282, 2005.

[85] Y. Sucu and N. Ünal, "Exact solution of Dirac equation in 2+1 dimensional gravity," Journal of Mathematical Physics, vol. 48, no. 5, Article ID 052503, 2007.

[86] A. Kempf, G. Mangano, and R. B. Mann, "Hilbert space representation of the minimal length uncertainty relation," Physical Review D: Particles, Fields, Gravitation and Cosmology, vol. 52, no. 2, pp. 1108-1118, 1995.

[87] S. Hossenfelder, M. Bleicher, S. Hofmann, J. Ruppert, S. Scherer, and H. Stöcker, "Signatures in the Planck regime," Physics Letters $B$, vol. 575, no. 1-2, pp. 85-99, 2003. 
[88] Z. W. Feng, H. L. Li, X. T. Zu, and S. Z. Yang, "Quantum corrections to the thermodynamics of Schwarzschild-Tangherlini black hole and the generalized uncertainty principle," The European Physical Journal C, vol. 76, article 212, 2016.

[89] Z. H. Li and L. M. Zhang, "Fermions tunnelling from black string and Kerr AdS black hole with consideration of quantum gravity," International Journal of Theoretical Physics, vol. 55, no. 1, pp. 401-411, 2016.

[90] S. Deser, R. Jackiw, and S. Templeton, "Three-dimensional massive gauge theories," Physical Review Letters, vol. 48, no. 15, pp. 975-978, 1982.

[91] S. Deser, R. Jackiw, and G. 't Hooft, “Three-dimensional Einstein gravity: dynamics of flat space," Annals of Physics, vol. 152, no. 1, pp. 220-235, 1984.

[92] E. Witten, " $2+1$ dimensional gravity as an exactly soluble system," Nuclear Physics B, vol. 311, no. 1, pp. 46-78, 1988.

[93] M. Banados, C. Teitelboim, and J. Zanelli, "Black hole in threedimensional spacetime," Physical Review Letters, vol. 69, no. 13, pp. 1849-1851, 1992.

[94] E. A. Bergshoeff, O. Hohm, and P. K. Townsend, "Massive gravity in three dimensions," Physical Review Letters, vol. 102, no. 20, Article ID 201301, 2009.

[95] H. Ahmedov and A. N. Aliev, "Type D solutions of 3D new massive gravity," Physical Review D: Particles, Fields, Gravitation and Cosmology, vol. 83, no. 8, 2011.

[96] I. Bakas and C. Sourdis, "Homogeneous vacua of (generalized) new massive gravity," Classical and Quantum Gravity, vol. 28, no. 1, Article ID 015012, 2011.

[97] J. Oliva, D. Tempo, and R. Troncoso, “Three-dimensional black holes, gravitational solitons, kinks and wormholes for BHT massive gravity," Journal of High Energy Physics, vol. 2009, no. 07, article 011, 2009.

[98] J. Oliva, D. Tempo, and R. Troncoso, "Static spherically symmetric solutions for conformal gravity in three dimensions," International Journal of Modern Physics A, vol. 24, no. 08n09, article 1588, 2009.

[99] Y. Kwon, S. Nam, J.-D. Park, and S.-H. Yi, “Quasi-normal modes for new type black holes in new massive gravity," Classical and Quantum Gravity, vol. 28, no. 14, Article ID 145006, 2011.

[100] P. A. Gonzalez and Y. Vazquez, "Dirac quasinormal modes of new type black holes in new massive gravity," European Physical Journal C, vol. 74, article 2969, 2014.

[101] R.-G. Cai, L.-M. Cao, and N. Ohta, "Thermodynamics of black holes in Hořava-Lifshitz gravity," Physics Letters B, vol. 5, pp. 504-509, 679.

[102] M. Dehghani, "Thermodynamics of (2+1)-dimensional charged black holes with power-law Maxwell field," Physical Review D, vol. 94, Article ID 104071, 2016.

[103] S. H. Hendi, S. Panahiyan, and B. E. Panah, "Geometrical method for thermal instability of nonlinearly charged BTZ black holes," Advances in High Energy Physics, vol. 2015, Article ID 743086, pp. 1-12, 2015.

[104] Y.-G. Miao and Y.-M. Wu, "Thermodynamics of the Schwarzschild-AdS black hole with a minimal length," Advances in High Energy Physics, vol. 2017, Article ID 1095217, pp. 1-14, 2017. 

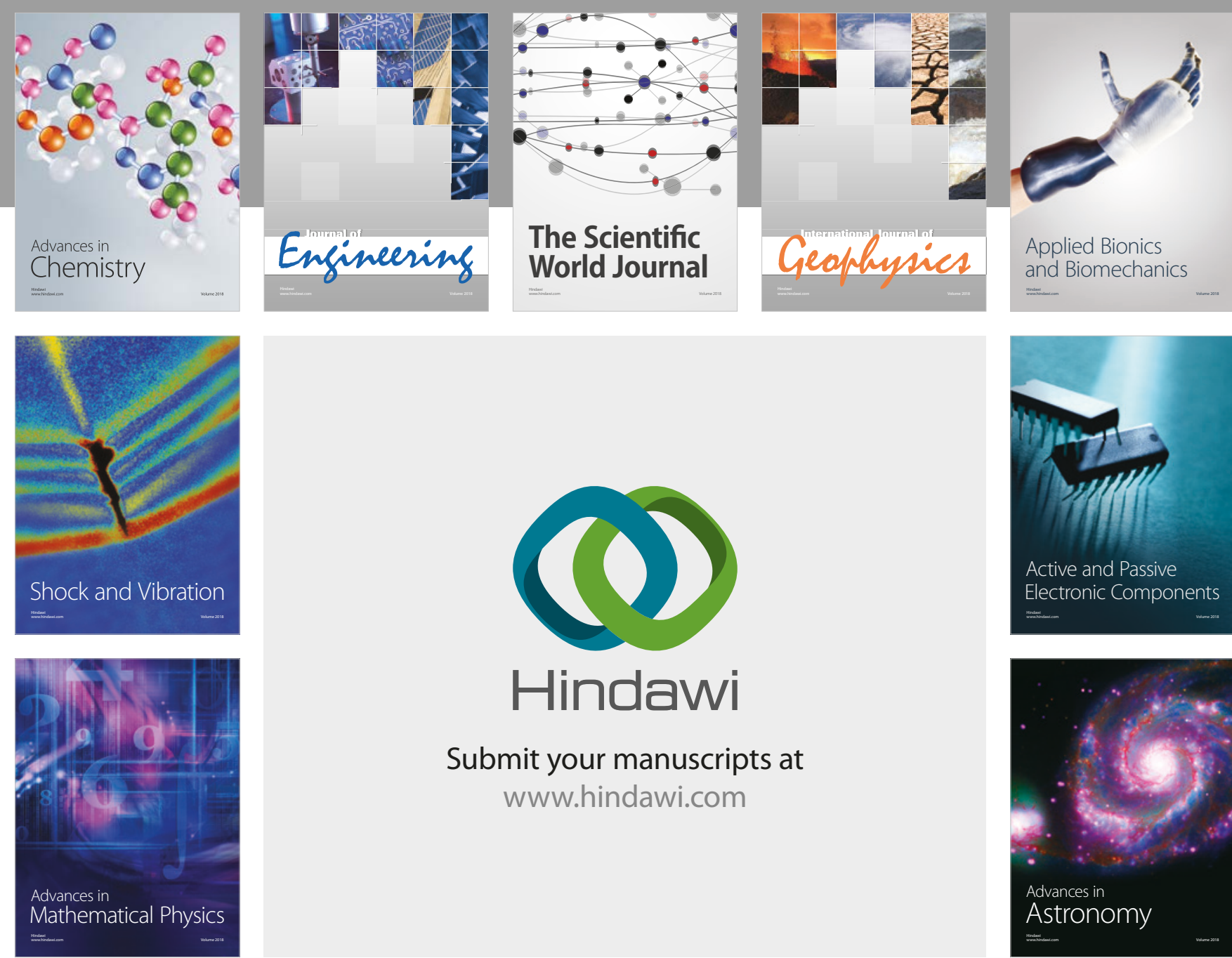

Submit your manuscripts at

www.hindawi.com

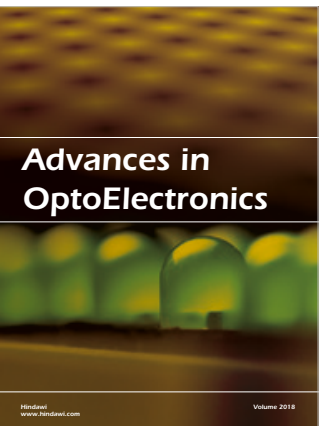

\section{Rotcting Machinery}
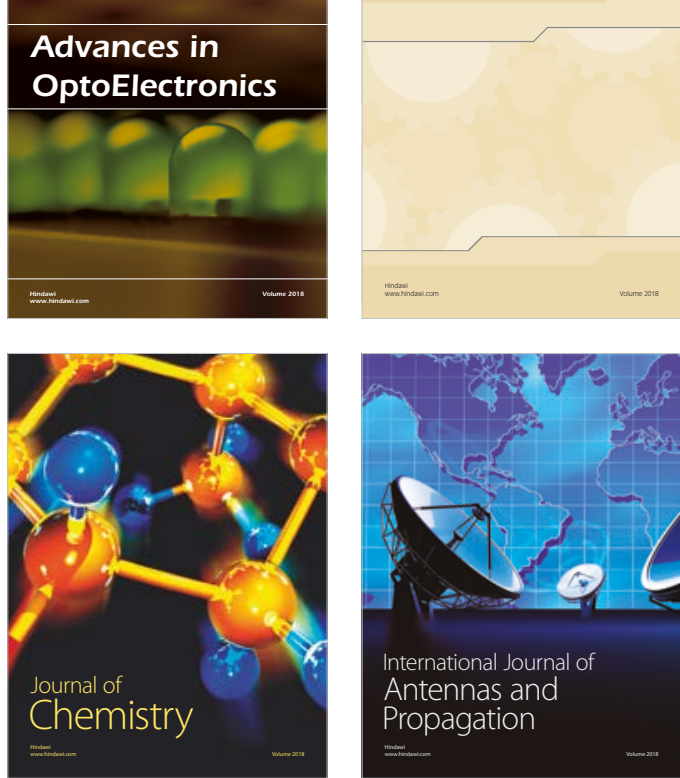

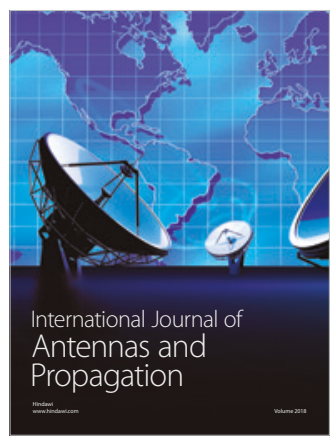

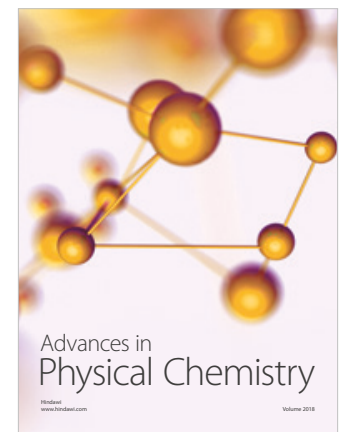

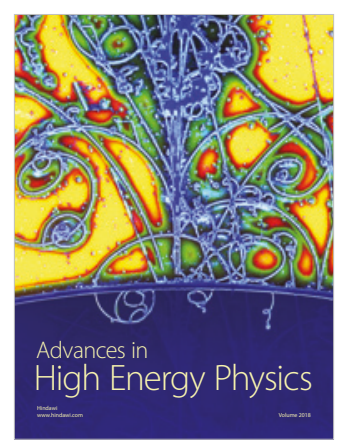

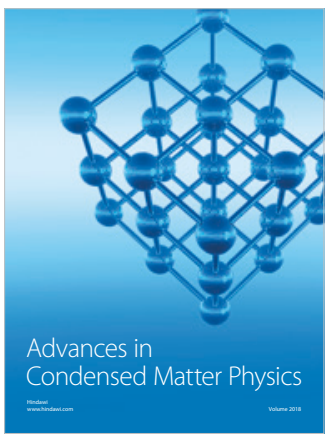

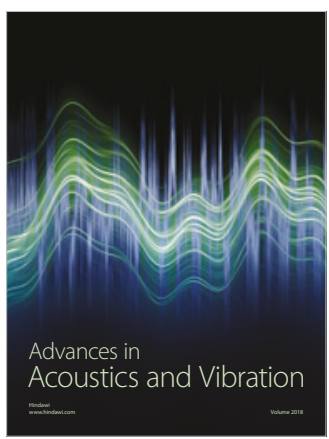

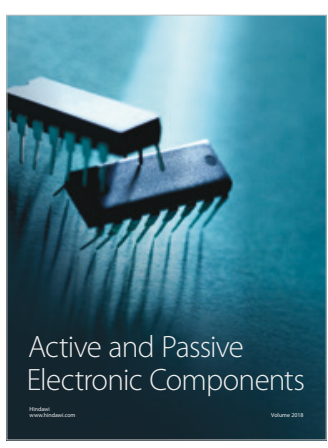
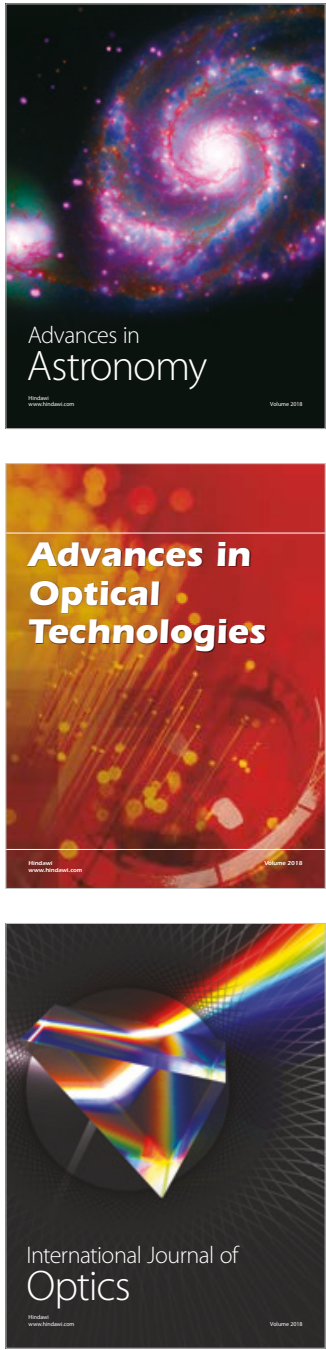